\title{
Management Challenge of Rhino-Orbito-Cerebral Mucormycosis in Covid 19 Era: A Prospective Observational Study
}

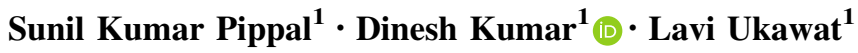

Received: 9 September 2021 / Accepted: 2 October 2021 / Published online: 23 October 2021

(C) Association of Otolaryngologists of India 2021

\begin{abstract}
Mucormycosis is an uncommon but a fatal fungal infection that usually affects patients with altered immunity. The Rhizopus Oryzae is most common type and responsible for nearly $60 \%$ of mucormycosis cases in humans and also accounts for $90 \%$ of the Rhino-orbitalcerebral (ROCM) form. Our aim and objective was to study, the site of mucormycosis in nose and paranasal sinuses, adjacent site like orbit palate or intracranial involvement, co-morbid condition and management to be done in confirm mucormycosis patients. The most common sinuses involved are the ethmoid, maxillary followed by the frontal and sphenoid sinus. Diabetes mellitus is often associated with mucormycosis of the paranasal sinuses, as is corona virus infection; uncontrolled diabetes further increases the risk. Intra-orbital involvement is common, but intracranial involvement is rare. Extensive steroid and broad-spectrum antibiotic use for Covid-19 management may cause or exacerbate fungal disease. All the patients required surgical intervention along with medical treatment.
\end{abstract}

Keywords Mucormycosis · Rhino-Orbital-Cerebral · Diabetes Mellitus

Dinesh Kumar

dineshjain2k7@gmail.com

1 Department of E.N.T., Bundelkhand Medical College, Sagar, M.P, India

\section{Introduction}

Corona virus disease 2019 (COVID-19) caused by severe acute respiratory syndrome corona virus 2 (SARS-CoV-2) has been associated with a wide range of opportunistic bacterial and fungal infections [1]. Both Aspergillosis and Candida have been reported as the main fungal pathogens for co-infection in people with COVID-19 [2].

Phycomycosis or zygomycosis was first described in 1885 by Paltauf [3] and later coined as Mucormycosis in 1957 by Baker [4] an American pathologist for an aggressive infection caused by Rhizopus. Mucormycosis is an uncommon but a fatal fungal infection that usually affects patients with altered immunity. Mucormycosis is an angioinvasive disease caused by mold fungi of the genus Rhizopus, Mucor, Rhizomucor, Cunninghamella and Absidia of Order- Mucorales, Class- Zygomycetes [5]. The Rhizopus Oryzae is most common type and responsible for nearly $60 \%$ of mucormycosis cases in humans and also accounts for $90 \%$ of the Rhino-orbital-cerebral (ROCM) form [6]. Mode of contamination occurs through the inhalation of fungal spores. Recently, several cases of mucormycosis in people with COVID19 have been increasingly reported world-wide, in particular from India.

The primary reason that appears to be facilitating Mucorales spores to germinate in people with COVID-19 is an ideal environment of low oxygen (hypoxia), high glucose (diabetes, new-onset hyperglycemia, steroid-induced hyperglycemia), acidic medium (metabolic acidosis, diabetic ketoacidosis [DKA]), high iron levels (increased ferritins) and decreased phagocytic activity of white blood cells (WBC) due to immunosuppression (SARS-CoV-2 mediated, steroid-mediated or background comorbidities) coupled with several other shared risk factors including 
prolonged hospitalization with or without mechanical ventilators.

Here, we present our recent and still ongoing experience of 80 cases of mucormycosis over a time period of just four months during second wave of covid 19, in these patients we studied involvement sinuses, adjacent site, different comorbid condition and management to be done.

\section{Aim and Objective}

was to study, the site of mucormycosis in nose and paranasal sinuses, adjacent site like orbit palate or intracranial involvement, co-morbid condition and management to be done in confirm mucormycosis patients.

\section{Inclusion Criteria}

(1) All confirm patient of mucormycosis during covid 19 irrespective of age and sex.

(2) Mucormycosis with co morbid condition like diabetes mellitus, chronic kidney disease.

\section{Exclusion Criteria}

(1) Patients having previous history of chronic rhinosinusitis with nasal polyposis before covid 19 .

\section{Materials and Method}

A prospective observational study was undertaken at Govt. Bundelkhand Medical College and Hospital, Sagar M.P, India, over a period of three months, from May to August 2021. All patients with invasive mucormycosis of the paranasal sinuses who presented to the ENT department, either as an out-patient or following departmental referral, and who were either corona virus-positive or had recovered from corona virus infection, were included in the study. The patients' presentation details, imaging findings, comorbidities, management details, and follow-up information were obtained, recorded and analyzed. Diagnostic nasal endoscopy of all the patients was done and black necrotic tissue was send to microbiology department for $\mathrm{KOH}$ mount and fungal culture. About 350 suspected patients were visited in our department whose diagnostic nasal endoscopy was done. Only 80 patients were proven as mucormycosis. Patients were admitted in separate mucor ward where whole team (including ENT surgeon, Eye surgeon, dental surgeon and general physician) visit and monitoring to be done. All patients were operated upon, keeping complete surgical debridement as the aim, along with intravenous amphotericin as well as posaconazole administration. Blood investigation (kidney function test, electrolyte, $\mathrm{C}$ - reactive protein) every 3rd day and Radiological investigation (CT OR MRI scan) done at $10^{\text {th }}$ postoperative day to see recurrence or extension of disease.

\section{Results}

Out of 350 suspected patient only 80 patients were proven as mucormycosis. A total of 80 patients presented; $68.75 \%$ (55 out of 80 ) of these were male and $31.25 \%$ (25 out of 80) were female. 10 of the patients were corona viruspositive at the time of presentation but had been infected for more than 14 days; $16.25 \%$ (13 out of 80 ) patients had no history of infection. All patients had a primary disease infection involving the ethmoid and maxillary sinus. Sphenoid and frontal involvement was less common (Fig. 1 and Table 1).

Out of the 80 patients, 30 had involvement of the eye at the time of presentation (Figs. 2 and 3, and Table 2); 15 had any vision loss. 06 patients gave consent for orbital exenteration. 15 of these patients were receiving intra-orbital amphotericin treatment. Intracranial involvement was seen very rarely, only in ten cases. The classical black eschar on the hard palate was observed in 34 patients. Seventy two of the patients were diabetic; 50 of these had uncontrolled blood sugar levels with hemoglobin A1c levels higher than 6.5 per cent, and the remaining 22 patients had controlled diabetes (Table 3). Sixty patients had hypertension; all of these were diabetic. One patient was in renal failure (chronic kidney disease) at the time of presentation. All 67 patients had used steroids during the management of their corona virus-associated illness. In all the patients' surgical intervention done FESS with debriment 58 patients, orbital exenteration 06, orbital decompression 10, maxillectomy 27, operated outside 12 patients, 10 patients required readmission (Fig. 4 and Table 4).

\section{Discussion}

Globally, the prevalence of mucormycosis varied from 0.005 to 1.7 per million populations, while its prevalence is nearly 80 times higher $(0.14$ per 1000$)$ in India compared to developed countries, in a recent estimate of year 2019-2020 [7-9]. In other words, India has highest cases of the mucormycosis in the world. Notwithstanding, India is already having second largest population with diabetes mellitus (DM) and was the diabetes capital of the world, until recently [10]. Importantly, DM has been the most common risk factor linked with mucormycosis in India, although hematological malignancies and organ transplant 
Fig. 1 Showing involvement of different sinuses
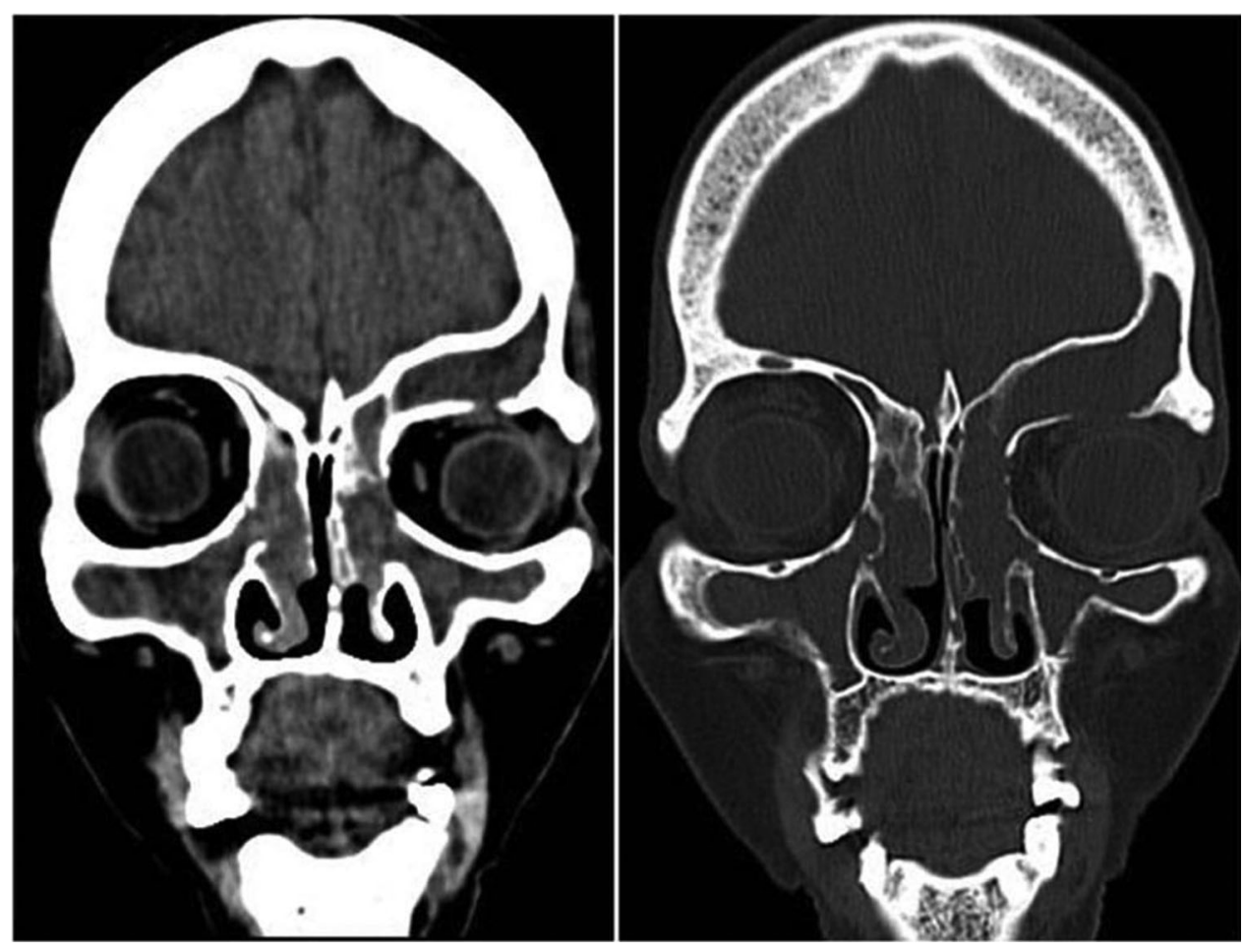

Table 1 Incidence of sinus affected

\begin{tabular}{llr}
\hline S. N & Sinus & Cases $(\mathrm{n} \%)$ \\
\hline 1 & Ethmoids & $80(100)$ \\
2 & Maxillary & $80(100)$ \\
3 & Frontal & $10(12.5)$ \\
4 & Sphenoid & $06(7.5)$ \\
\hline
\end{tabular}
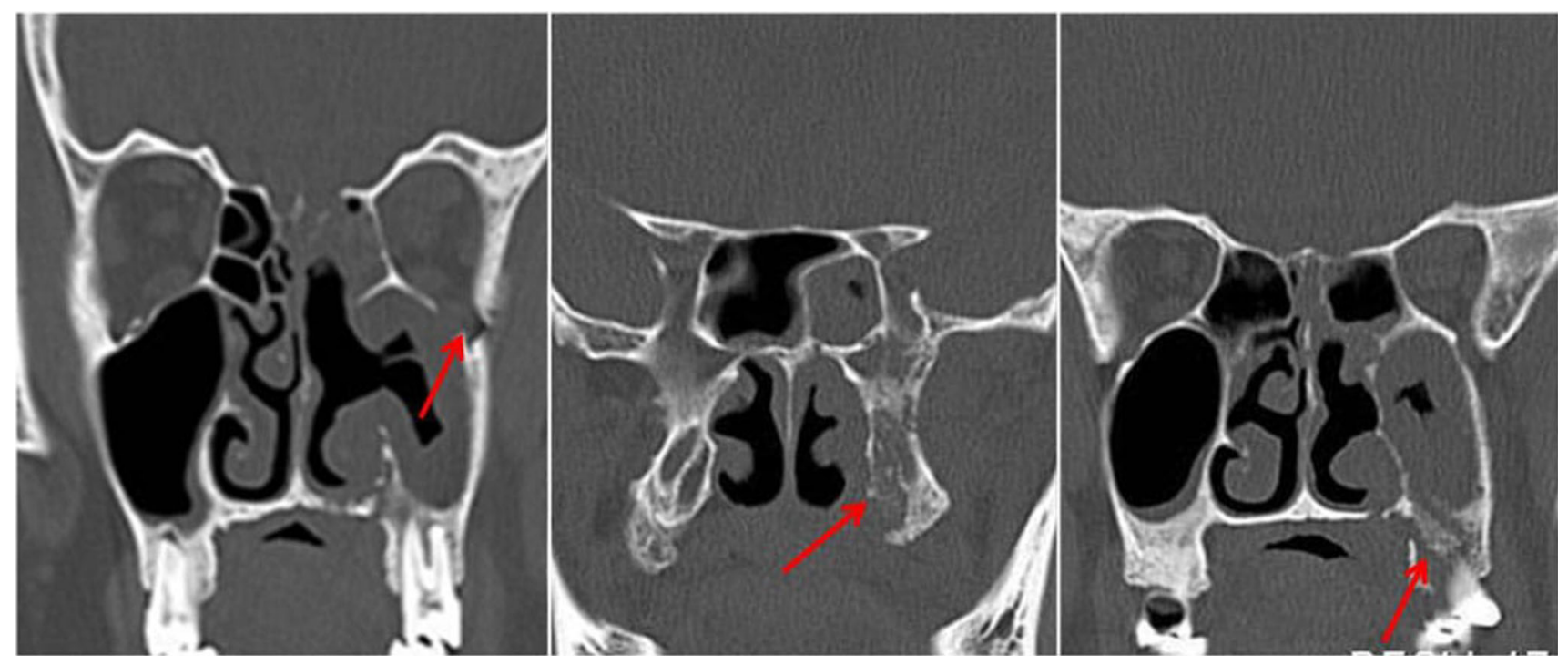

Fig. 2 showing involvement of adjacent site 
Fig. 3 Associated co-morbid conditions

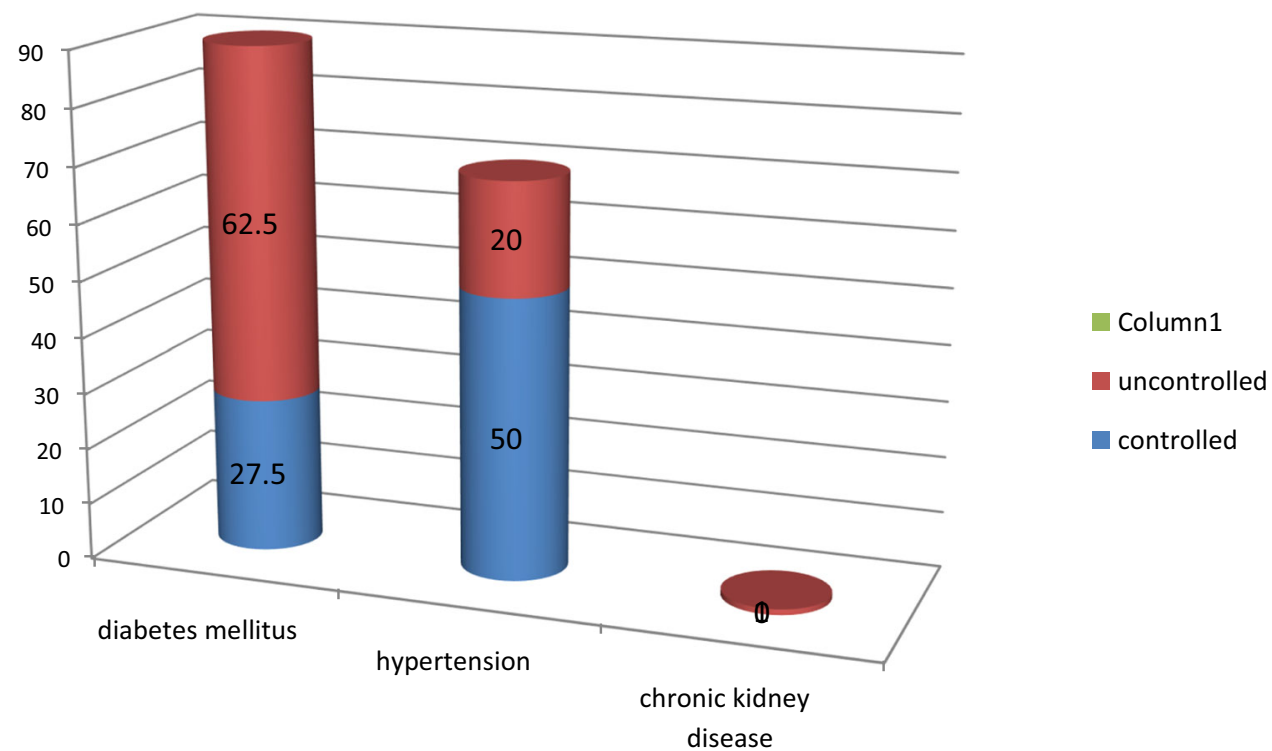

Table 2 Spread of infection to adjacent sites at time of presentation

\begin{tabular}{llr}
\hline S. N & Adjacent spread & Cases $(\mathrm{n} \%)$ \\
\hline 1 & Intraorbital & $30(37.5)$ \\
2 & Palate & $34(42.5)$ \\
3 & Intracranial & $10(12.5)$ \\
\hline
\end{tabular}

Table 3 Associated co-morbid conditions

\begin{tabular}{llll}
\hline S. N & Co-morbidity & Status & Cases $(\mathrm{n} \%)$ \\
\hline 1 & Diabetes mellitus & Controlled & $22(27.5)$ \\
& & Uncontrolled & $50(62.5)$ \\
2 & Hypertension & Controlled & $40(50)$ \\
& & Uncontrolled & $20(25)$ \\
3 & Others & Chronic kidney diseases & $01(1.25)$ \\
\hline
\end{tabular}

takes the lead in Europe and the USA [9]. Nevertheless, DM remains the leading risk factor associated with mucormycosis globally, with an overall mortality of $46 \%$ [11]. Indeed, presence of DM was an independent risk factor (Odds ratio [OR] 2.69; 95\% Confidence Interval $1.77-3.54 ; P<0.001)$ in a large 2018 meta-analysis of 851 cases of rarely occurring mucormycosis, and the most common species isolated was Rhizopus (48\%) [11]. While long term use of corticosteroids has often been associated with several opportunistic fungal infection including Aspergillosis and mucormycosis, even a short course of corticosteroids has recently been reported to link with mucormycosis especially in people with DM. A cumulative prednisone dose of greater than $600 \mathrm{mg}$ or a total methyl prednisone dose of 2-7 g given during the month before predisposes immunocompromised people to mucormycosis [12]. There are few case reports of mucormycosis resulting from even a short course (5-14 days) of steroid therapy, especially in people with DM [13]. Surprisingly, $46 \%$ of the patients had received corticosteroids within the month before the diagnosis of mucormycosis in the European Confederation of Medical Mycology study [14]. Especially the intracranial involvement of mucormycosis increases the fatality rate to as high as $90 \%$ [15].

Mucormycosis is characterized by the presence of hyphae invasion of sinus tissue and a time course of less 
Fig. 4 Distribution of patients managed surgically

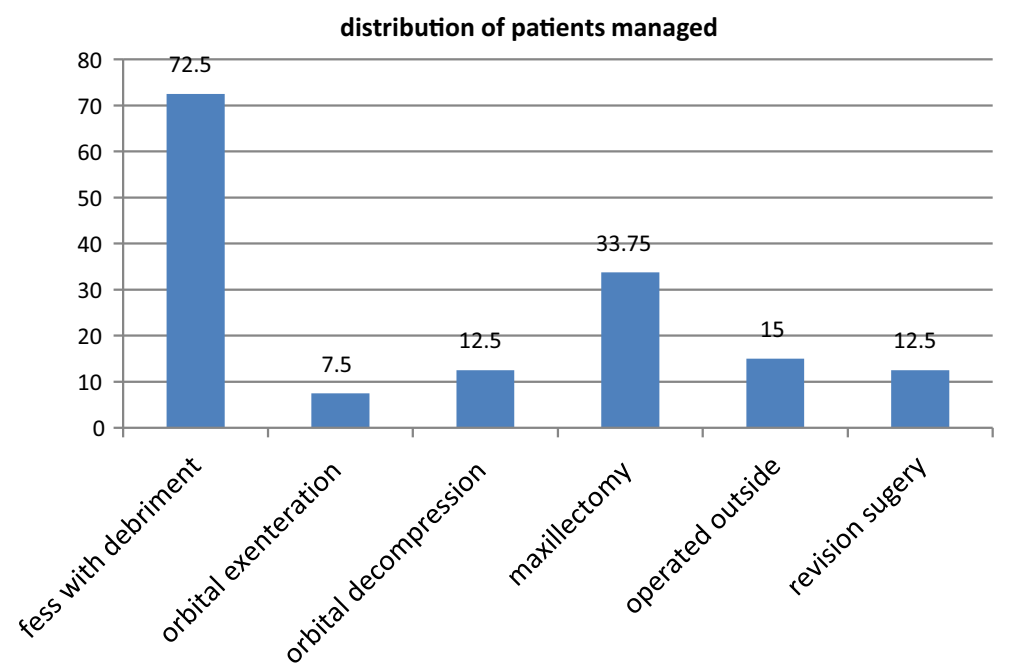

no of patient (\%)

Table 4 Distribution of patients managed surgically

\begin{tabular}{lll}
\hline S. N & Surgical procedure & No of patients (n \%) \\
\hline 1 & FESS with debriment & 58 \\
2 & Orbital exenteration & 06 \\
3 & Orbital decompression & 10 \\
4 & Maxillectomy & 27 \\
5 & Operated outside & 12 \\
6 & Patient readmitted for revision surgery & 10 \\
\hline
\end{tabular}

than four weeks [16, 17]. Clinically, rhinocerebral mucormycosis can present with atypical signs and symptoms similar to complicated sinusitis, such as nasal blockade, crusting, proptosis, facial pain and oedema, ptosis, chemosis, and even ophthalmoplegia, with headache and fever and various neurological signs and symptoms if intracranial extension is present [18, 19]. A black eschar is often seen in the nasal cavity or over the hard palate region, but is not characteristic [20, 21]. Histological features include mycotic infiltration of blood vessels, vasculitis with thrombosis, tissue infarction, haemorrhage and acute neutrophilic infiltrate [21]. Without early diagnosis and treatment, there may be rapid progression of the disease, with reported mortality rates from intra-orbital and intracranial complications of 50-80 per cent [22]. Even with prompt diagnosis, treatment of underlying diseases, and aggressive medical and surgical intervention, the management is often not effective, leading to an extension of the infection and ultimately death [23].

Covid-19 is a life-threatening, infectious disease, affected patients show an over expression of inflammatory cytokines, and impaired cell-mediated immunity with decreased cluster of differentiation 4 and 8 positive T-helper $(\mathrm{CD} 4+\mathrm{T}$ and $\mathrm{CD} 8+\mathrm{T})$ cell counts, indicating susceptibility to fungal co-infections [24]. Critically ill patients, especially those admitted to intensive care units and those who required mechanical ventilation, or who had a longer duration of hospital stays, even as long as 50 days, were more likely to develop fungal co-infections [25]. Extensive use of steroids in Covid-19 management can also suppress immunity, allowing opportunistic fungal infections to colonize. Hence, it is important to be aware that Covid-19 patients can develop further fungal infections during the middle and latter stages of this disease, especially severely ill individuals [26].

The Covid-19 infection caused by the novel SARSCoV-2 has been associated with a wide range of disease patterns, ranging from a mild cough to life-threatening pneumonia [27]. Besides the diffuse alveolar damage with severe inflammatory exudation, Covid-19 patients always have immunosuppression with a decrease in CD4 $+\mathrm{T}$ and $\mathrm{CD} 8+\mathrm{T}$ cells [28]. During the SARS-CoV infection spread in 2003, the incidence of fungal infection was 14.8-27 per cent, and it was them a in cause of death for severe acute respiratory syndrome patients, accounting for 25-73.7 percent in all causes of death [29-31]. Studies have shown that SARS-CoV and SARS-CoV-2 belong to the same species, and have similar prevalence rates and 
biological and clinical characteristics [32]. Based on our experience in 2003, it is important that physicians pay critical attention to the high probability of increased incidence of fungal infections in Covid-19 affected or recovered patients, similar to the finding observed in mucormycosis cases here. Previously, few such incidental case reports have been published, but a firm association between Covid-19 and increased fungal infections can now be clearly seen. Mehta and Pandey reported a single case of a 60-year-old male with rhino-orbital mucormycosis associated with Covid-19 in September 2020 [27]. another such case report was published by Werthman-Ehrenreich in the same month [33]. White et al. studied 135 adults with Covid-19 infection, and reported an incidence of 26.7 per cent for invasive fungal infections [34]. Song et al. studied the association between Covid-19 and invasive fungal sinusitis in April 2020, and concluded that a large number of patients affected by or recovered from Covid-19 are at increased risk of developing invasive fungal diseases, and gave a management algorithm for such cases [35]. In a recent review, 8 per cent of corona virus-positive or recovered patients had secondary bacterial or fungal infections during hospital admission, with widespread use of broad-spectrum antibiotics and steroids [25]. There are various possible reasons for this association, including the immunosuppression caused by Covid-19 infection and disease process, or the extensive use of steroids and broadspectrum antibiotics in the management of Covid-19, leading to the development or exacerbation of a pre-existing fungal disease. The National Institute of Health, according to the Randomised Evaluation of Covid-19 Therapy ('RECOVERY') Collaborative Group, recommends steroid use only in patients who are on a ventilator or require supplemental oxygen, but not in milder cases [36]. The guidelines specifically mention the risk of developing a secondary infection. [37] Mucor is a saprophytic fungus; its spores exist widely in nature, and are spread in soil, air, food and decaying organic material [23]. Because of the low virulence potential, it may be present in the nasal mucosa of healthy people as a commensal [38]. If the patient becomes immunosuppressed, this fungus may germinate within the paranasal sinuses, and spread intracranially or to other nearby structures such as the orbit. Non-contrast computed tomography of the paranasal sinuses is usually the first investigation of choice, with gadolinium-enhanced magnetic resonance imaging being resorted to if intra-orbital or intracranial extension is suspected. Focal bony erosions and extra-sinus spread are strongly suggestive of the diagnosis [23]. Surgical debridement of the infected area should be performed as soon as possible once the diagnosis is confirmed. Surgery alone has been reported not to be curative, but an aggressive surgical approach has been shown to improve survival
[39, 40]. Amphotericin-B deoxycholate remains the antifungal treatment of choice to start, with its liposomal preparations preferred because of decreased nephrotoxicity. In cases refractory or intolerant to amphotericin therapy, posaconazole is considered a suitable alternative option [17, 40]. Prognosis remains poor even with aggressive surgery and intravenous anti-fungal therapy, with reported mortality rates of $33.3-80$ per cent, going up to 100 per cent in disseminated infections [17, 41]. We found 80 such cases of mucormycosis of the paranasal sinuses over the four-month study period; maximum no of these patients were either corona virus-positive at the time of diagnosis or had been infected previously only few patients had no history of covid infection. All patients underwent surgical debridement. All patients were follow up had received up to four months' follow up by the end of the study period. There were no mortalities.

\section{Conclusion}

Mucormycosis is a fungal infection with high mortality and rising incidence mostly associated with corona virus disease 2019 (Covid-19) affected or recovered patients. The most common sinuses involved are the ethmoid, maxillary followed by the frontal and sphenoid sinus. Diabetes mellitus is often associated with mucormycosis of the paranasal sinuses, as is corona virus infection; uncontrolled diabetes further increases the risk. Intra-orbital involvement is common, but intracranial involvement is rare. Extensive steroid and broad-spectrum antibiotic use for Covid-19 management may cause or exacerbate fungal disease. All the patients required surgical intervention along with medical treatment.

\section{Declarations}

Conflict of Interest Dr Sunil kumar Pippal (Professor and Head) declares that there is no conflict of interest. Dr Dinesh Kumar declares that there is no conflict of interest. Dr Lavi Ukawat declares that there is no conflict of interest.

Consent for Publication Proper consent were taken.

\section{References}

1. Kubin CJ, McConville TH, Dietz D et al (2021) Characterization of bacterial and fungal infections in hospitalized patients with COVID-19 and factors associated with healthcare-associated infections. Open Forum Infect Dis 8(6):ofab201. https://doi .org/10.1093/ofid/ofab201

2. Song G, Liang G, Liu W (2020) Fungal co-infections associated with global COVID19 pandemic: a clinical and diagnostic perspective from China. Mycopathologia 185(4):599-606 
3. Paltauf A (1885) Mycosis mucorina. Virchows Arch Pathol Anat Physiol Klin Med 102:543-564

4. Baker RD (1957) Mucormycosis-a new disease? J Am Med Assoc 163:805-808

5. Eucker J, Sezer O, Graf B, Possinger K (2001) Mucormycoses. Mycoses 44(7):253-260

6. Sugar AM (2000) In: Mandell GL, Bennett JE, Dolin R(eds) Mandell, Douglas, and Bennett's principles and practice of infectious diseases, 5th edn. Churchill Livingstone, New York, USA

7. Skiada A, Pavleas I, Drogari-Apiranthitou M (2020) Epidemiology and diagnosis of mucormycosis: an update. J Fungi 6(4):265

8. Chander J, Kaur M, Singla N et al (2018) Mucormycosis: battle with the deadly enemy over a five-year period in India. J Fungi 4(2):46. https://doi.org/10.3390/jof 4020046

9. Prakash H, Chakrabarti A (2019) Global epidemiology of mucormycosis. J Fungi 5:26

10. International Diabetes Federation. Idf Diabetes Atlas. 2019. Available online: https://diabetesatlas.org/en/resources/ (accessed on May 10, 2021).

11. Jeong W, Keighley C, Wolfe R et al (2019) The epidemiology and clinical manifestations of mucormycosis: a systematic review and meta-analysis of case reports. Clin Microbiol Infect 25:26-34

12. Lionakis MS, Kontoyiannis DP (2003) Glucocorticoids and invasive fungal infections. Lancet 362:1828-1838

13. Hoang K, Abdo T, Reinersman JM, Lu R, Higuita NIA (2020) A case of invasive pulmonary mucormycosis resulting from short courses of corticosteroids in a wellcontrolled diabetic patient. Med Mycol Case Rep 29(1):22-24

14. Skiada A, Pagano L, Groll A et al (2011) Zygomycosis in Europe: analysis of 230 cases accrued by the registry of the European confederation of medical mycology (ECMM) working group on zygomycosis between 2005 and 2007. Clin Microbiol Infect 17(12):1859-1867

15. Deutsch PG, Whittaker J, Prasad S (2019) Invasive and noninvasive fungal rhinosinusitis - a review and update of the evidence. Medicina 55:1-14

16. Ferguson BJ (2000) Definitions of fungal rhinosinusitis. Otolaryngol Clin North Am 33:227-235

17. Chakrabarti A, Denning DW, Ferguson BJ, Ponikau J, Buzina W, Kita $\mathrm{H}$ et al (2009) Fungal rhinosinusitis: a categorization and definitional schema addressing current controversies. Laryngoscope 119:1809-1818

18. Scheckenbach K, Cornely O, Hoffmann TK, Engers R, Bier H, Chaker A et al (2010) Emerging therapeutic options in fulminant invasive rhinocerebral mucormycosis. Auris Nasus Larynx 37:322-328

19. Vairaktaris E, Moschos MM, Vassiliou S, Baltatzis S, Kalimeras E, Avgoustidis D et al (2009) Orbital cellulitis, orbital subperiosteal and intraorbital abscess. Report of three cases and review of the literature. J Craniomaxillofac Surg 37(3):132-136

20. Mohindra S, Mohindra S, Gupta R, Bakshi J, Gupta SK (2007) Rhinocerebral mucormycosis: the disease spectrum in 27 patients. Mycoses 50:290-296

21. Munir N, Jones NS (2007) Rhinocerebral mucormycosis with orbital and intracranial extension: a case report and review of optimum management. J Laryngol Otol 121:192-195

22. DeShazo RD, Chapin K, Swain RE (1997) Fungal sinusitis. N Engl J Med 337:254-259

23. Gillespie MB, O'Malley BW (2000) An algorithmic approach to the diagnosis and management of invasive fungal rhinosinusitis in the immunocompromised patient. Otolaryngol Clin North Am 33:323-334

24. Ballester DG, González-García R, García CM, Ruiz-Laza L, Gil FM (2012) Mucormycosis of the head and neck: report of five cases with different presentations. J Craniomaxillofac Surg 40:584-591

25. Song G, Liang G, Liu W (2020) Fungal co-infections associated with global COVID-19 pandemic: a clinical and diagnostic perspective from China. Mycopathologia 185:599-606

26. Yang X, Yu Y, Xu J, Shu H, Liu H, Wu Y et al (2020) Clinical course and outcomes of critically ill patients with SARS-CoV-2 pneumonia in Wuhan, China: a single-centered, retrospective, observational study. Lancet Respir Med 8:475-481

27. Gangneux JP, Bougnoux ME, Dannaoui E, Cornet M, Ralph ZJ (2020) Invasive fungal diseases during COVID-19: we should be prepared. J Mycol Med 30:100971

28. Mehta S, Pandey A (2020) Rhino-orbital mucormycosis associated with COVID-19. Cureus 12:e10726

29. Yang W, Cao Q, Qin L, Wang X, Cheng Z, Pan A et al (2020) Clinical characteristics and imaging manifestations of the 2019 novel coronavirus disease (COVID-19): a multi-center study in Wenzhou city, Zhejiang. China J Infect 80:388-393

30. Zhang Y, Li WX, Huang KW, Cao ZX, Hao JY (2003) Hospital acquired pneumonia occurring after acute stage of the serious SARS and its treating strategies. Chin J Nosocomiol 11:1081-1087

31. Yin CH, Wang C, Tang Z, Zhang SW, Wang BS (2004) Clinical analysis of 146 patients with critical severe acute respiratory syndrome in Beijing areas. Clin J Emerg Med 1:12-14

32. Li CS, Pan SF (2003) Analysis and causation discussion of 185 severe acute respiratory syndrome dead cases [in Chinese]. Zhongguo Wei Zhong Bing Ji Jiu Yi Xue 15:582-584

33. Peeri NC, Shrestha N, Rahman MS, Zaki R, Tan Z, Bibi S et al (2020) The SARS, MERS and novel coronavirus (COVID-19) epidemics, the newest and biggest global health threats: what lessons have we learned? Int J Epidemiol 49:717-726

34. Werthman-Ehrenreich A (2021) Mucormycosis with orbital compartment syndrome in a patient with COVID-19. Am J Emerg Med 42:264.e5-264.e8

35. White L, Dhillon R, Cordey A, Hughes H, Faggian F, Soni S et al (2020) A national strategy to diagnose coronavirus disease 2019 associated invasive fungal disease in the intensive care unit. Clin Infect Dis 73(7):ciaa1298

36. Rawson TM, Moore LS, Zhu N, Ranganathan N, Skolimowska K, Gilchrist $M$ et al (2020) Bacterial and fungal coinfection in individuals with coronavirus: a rapid review to support COVID19 antimicrobial prescribing. Clin Infect Dis 71:2459-2468

37. RECOVERY Collaborative Group, Horby P, Lim WS, Emberson JR, Mafham M, Bell JL et al (2021) Dexamethasone in hospitalized patients with Covid-19-preliminary report. N Engl J Med 384:693-704

38. National Institutes of Health. Coronavirus disease 2019 (COVID19) treatment guidelines. In: https://www.covid19treatmentguidelines.nih.gov/ [15 July 2020]

39. Elinav H, Zimhony O, Cohen MJ, Marcovich AL, Benenson S (2009) Rhinocerebral mucormycosis in patients without predisposing medical conditions: a review of the literature. Clin Microbiol Infect 15:693-697

40. Goldstein EJ, Spellberg B, Walsh TJ, Kontoyiannis DP, Edwards J Jr, Ibrahim AS (2009) Recent advances in the management of mucormycosis: from bench to bedside. Clin Infect Dis 48:1743-1751

41. Jung SH, Kim SW, Park CS, Song CE, Cho JH, Lee JH et al (2009) Rhinocerebral mucormycosis: consideration of prognostic factors and treatment modality. Auris Nasus Larynx 36:274-279

Publisher's Note Springer Nature remains neutral with regard to jurisdictional claims in published maps and institutional affiliations. 